\title{
ABSOLUTE RETRACTS IN GROUP THEORY
}

\section{REINHOLD BAER}

The subgroup $R$ of the group $G$ has been termed a retract ${ }^{1}$ of the group $G$ whenever there exists an idempotent endomorphism of $G$ which maps $G$ upon $R$. This definition is in strict analogy to the topological concept of retract. Thus one may be tempted to define absolute retracts in like similarity to topological usage. However, we shall prove in the course of the present note that the identity is the only group which is a retract of every containing group. Consequently only modifications of the topological concept will be useful, and we shall show that each of the following classes of groups may in a certain sense lay claim to the title of absolute retract: the complete groups, the abelian groups the orders of whose elements are finite and square free, and the free groups.

The following definition of the concept of retract is equivalent to the one given above, but it will be a little bit easier to handle: The group $R$ is a retract of the group $G$ if $R$ is a subgroup of $G$, and if there exists an endomorphism $\epsilon$ of $G$ with the following properties:

for $r$ in $R$.

$$
G^{\epsilon} \leqq R \text { and } r^{e}=r
$$

Complete groups (Carmichael [2, p. 81]) have been defined as groups with the following properties: each of their automorphisms is an inner automorphism and their center consists of the identity only. Thus groups are complete if they are "essentially" identical with their group of automorphisms.

THEOREM 1. The group $G$ is complete if, and only if, it meets the following requirement:

$\left(^{*}\right)$ If $G$ is a normal subgroup of the group $E$, then $G$ is a retract of $E$.

Proof. Assume first the validity of condition $\left({ }^{*}\right)$. Consider the automorphism $\alpha$ of $G$. Then there exists (Zassenhaus [1, pp. 93, 94]) one and essentially only one group $A$ which is obtained by adjoining to $G$ an element $t$ subject to the relations

$$
t^{-1} g t=g^{\alpha}
$$

for $g$ in $G$. Clearly $G$ is a normal subgroup of $A$ and $A / G$ is an infinite

Presented to the Society, April 27, 1946; received by the editors January 30, 1946.

${ }^{1}$ See Baer [2, chap. II, \$3] for elementary properties of retracts. Many applications of the concept of retract may be found there too. Numbers in brackets refer to the Bibliography at the end of the paper. 
cyclic group. Hence there exists by $\left(^{*}\right)$ an endomorphism $\beta$ of $A$ with the following properties:

$$
A^{\beta} \leqq G \text { and } g^{\beta}=g
$$

for $g$ in $G$. In particular $s=t^{\beta}$ is an element in $G$, and this element satisfies, for every element $g$ in $G$,

$$
s^{-1} g s=\left(t^{-1}\right)^{\beta} g^{\beta} t^{\beta}=\left(t^{-1} g t\right)^{\beta}=g^{\alpha \beta}=g^{\alpha},
$$

since $g$ and $g^{\alpha}$ belong to $G$. Thus the automorphism $\alpha$ is induced by the element $s$ in $G$, proving that every automorphism of $G$ is inner.

Consider next the element $z$ in the center of $G$. Then there exists (Zassenhaus $[1$, p. 96, Satz 21]) one and essentially only one group $Z$ which is obtained by adjoining to $G$ elements $x$ and $y$ subject to the following relations:

$$
x^{-1} y^{-1} x y=z, \quad x g=g x \text { and } y g=g y
$$

for every $g$ in $G$. Then $G$ is a normal subgroup of $Z$ and $Z / G$ is a free abelian group of rank 2 . Hence there exists by $\left({ }^{*}\right)$ an endomorphism $\epsilon$ of $Z$ with the following properties:

$$
Z^{\epsilon} \leqq G \text { and } g^{\epsilon}=g
$$

for every $g$ in $G$. If $g$ is an element in $G$, then

$$
x^{\epsilon} g=x^{\epsilon} g^{\epsilon}=(x g)^{\epsilon}=(g x)^{\epsilon}=g^{\epsilon} x^{\epsilon}=g x^{\epsilon},
$$

proving that $x^{e}$ belongs to the center of $G$, and likewise we show that $y^{*}$ belongs to the center of $G$. Consequently

$$
z=z^{e}=x^{-\epsilon} y^{-\epsilon} x^{e} y^{e}=1 \text {. }
$$

Thus we have shown that 1 is the only element in the center of $G$, and this completes the proof of the fact that every group with the property $(*)$ is complete.

Assume conversely that the group $G$ is complete and that $G$ is a normal subgroup of the group $T$. Denote by $S$ the centralizer of $G$ in $T$ (which consists of all those elements in $T$ which commute with every element in $G$ ). Since every automorphism of $G$ is an inner automorphism, it follows that $T=G S=S G$; and since the identity is the only element in the center of $G$, it follows that 1 is the cross cut of $G$ and $S$. Hence $T$ is the direct product (Carmichael $[1$, p. 96, no. 10]) of $G$ and $S$, and this fact clearly implies that $G$ is a retract of $T$, completing the proof.

Groups containing a given group $G$ as normal subgroups are termed (Zassenhaus $[1$, p. $89 \mathrm{ff}$.$] ) extensions of G$. It may be noted that in the 
first part of the proof of Theorem 1 we made use only of extensions of $G$ by abelian groups. This shows the validity of the following proposition:

COROLLARY 1. The group $G$ is complete if (and only if) it is a retract of every extension of $G$ by an abelian group.

REMARK. Examples of complete groups are provided by all the symmetric groups of finite or infinite degree, ${ }^{2}$ not less than 7 . This shows in particular that every group is a subgroup of a complete group and thus a subgroup of a group, meeting requirement $\left(^{*}\right)$ of Theorem 1.

THEOREM 2. The identity is the only group which is a retract of every containing group.

Proof. Suppose that the group $G$ is a retract of every group which contains $G$ as a subgroup. Denote by $D$ the direct product of $G$ and of a group $H$ isomorphic to $G$, and denote by $\eta$ a definite isomorphism of $G$ upon $H$. Denote by $W$ the essentially uniquely determined group (Zassenhaus $[1$, pp. 93, 94]) which is obtained by adjoining to $D$ an element $w$ subject to the relations:

$$
w^{2}=1, \quad w g w=g^{\eta}
$$

for $g$ in $G$. Then the element $w$ of order 2 induces in $D$ the automorphism which interchanges the elements $g$ and $g^{\eta}$ for $g$ in $G$. Clearly $D$ is a normal subgroup of $W$ and $W / D$ of order 2 . Since, therefore, $G$ is a subgroup of the group $W$, there exists an endomorphism $\delta$ of $W$ with the following properties:

$$
W^{\delta} \leqq G \text { and } g^{\delta}=g
$$

for $g$ in $G$. We note next that every element in $G$ commutes with every element in $H$. If $x$ is an element in $H$, and if $g$ is an element in $G$, then

$$
g x^{\delta}=g^{\delta} x^{\delta}=(g x)^{\delta}=(x g)^{\delta}=x^{\delta} g^{\delta}=x^{\delta} g,
$$

proving that $x^{\delta}$ is in the center of $G$, since $x^{\delta}$ belongs to $G$. Hence $H^{\delta}$ is part of the center of $G$.

If $g$ is any element in $G$, then $g^{\eta}$ is in $H$ and $g^{\eta \delta}$ belongs, therefore, to the center of $G$. Hence

$$
w^{\delta} g=w^{\delta} g^{\delta}=(w g)^{\delta}=\left(g^{\eta} w\right)^{\delta}=g^{\eta \delta} w^{\delta}=w^{\delta} g^{\eta \delta},
$$

since $w^{\delta}$ is in $G$ too. Consequently $g=g^{\eta \delta}$, proving that $G=H^{\delta}$. Thus

2 See Baer [1], Schreier and Ulam [1, 2]. 
$G$ is commutative, since we showed before that $H^{\delta}$ is part of the center of $G$.

But $G$ is a retract of each of its extensions, and thus it follows from Theorem 1 that the center of $G$ is 1 . Hence $G=1$, as we intended to prove.

REMARK. The situation would be changed considerably if we restricted the class of admissible groups. We mention in this respect the following well known theorem: The abelian group $A$ is a retract of every abelian group if, and only if, $A=A^{p}$ for every prime number $p$. On the other hand the preceding theorems would not be affected at all by restricting oneself to finite groups.

THEOREM 3. Every subgroup of the group $G$ is a retract of $G$ if, and only if, $G$ is an abelian group the orders of whose elements are finite and square free.

Proof. If $G$ is an abelian group the orders of whose elements are square free integers, then every subgroup of $G$ is known to be a direct factor of $G$. But direct factors are retracts, proving the sufficiency of our condition.

Assume conversely that every subgroup of $G$ is a retract of $G$. Then there exists to every element $g$ in $G$ an endomorphism $\epsilon$ of $G$ with the following properties: $g^{\epsilon}=g$ and $G^{\epsilon}$ is part of the cyclic subgroup generated by $g$.

Denote by $E$ the kernel of the endomorphism $\epsilon$. Then $G / E$ is a cyclic group. This implies in particular that the commutator subgroup of $G$ is part of $E$. If $g \neq 1$, then $g$ is certainly not in $E$. Thus it is impossible that an element, not 1 , is in the commutator subgroup of $G$. Consequently $G$ is abelian.

Consider an element $z$ in $G$ and a positive integer $n$. Then there exists an endomorphism $\nu$ of $G$ with the following properties: $\left(z^{n}\right)^{\nu}=z^{n}$ and $G^{v}$ is part of the cyclic subgroup generated by $z^{n}$.

The element $z$ itself is therefore mapped by $\nu$ upon a power of $z^{n}$. Hence there exists an integer $m=m(n, z)$ such that $z^{\nu}=z^{n m}$. Consequently we find that

$$
z^{n}=\left(z^{\nu}\right)^{n}=z^{n m n} \quad \text { or } \quad z^{n(1-m n)}=1 .
$$

Let in particular $n=2$. Then $1-2 m(2, z)$ is an odd number $r$ such that $z^{2 r}=1$, proving that $z$ is of finite order. Next we take for $n$ any divisor of the order of $z$. Then $n$ and $1-m n(n, z)$ are relatively prime numbers, and we infer from $z^{n(1-m n)}=1$ that the order of $z$ is square free. This completes the proof.

Dualizing the concept of retract of a group, we say that the quotient 
group $G / N$ is retractable whenever there exists an idempotent endomorphism of $G$ whose kernel is exactly $N$. This is equivalent to saying that $G$ is a splitting extension of $N$ by $G / N$. We note the following known theorem : ${ }^{3}$

The group $F$ is a free group if, and only if, every quotient group, isomorphic to $F$, is retractable.

This theorem may be considered a dual of either or both Theorems 1 and 2. In order to find a dual of Theorem 3 one has to determine the class of totally retractable groups. Here we term a group totally retractable if each of its quotient groups is retractable. A complete characterization of this class of groups does not seem to be available as yet. Thus the following criteria may be of interest.

THEOREM 4. The abelian group $A$ is totally retractable if, and only if, the orders of the elements in $A$ are finite and square free.

Proof. If the orders of the elements in $A$ are finite and square free, then every subgroup of $A$ is a direct factor of $A$. Consequently every quotient group is retractable and $A$ is totally retractable.

Assume conversely that $A$ is totally retractable. Denote by $F$ the subgroup $A$ which consists of the elements of finite order in $A$. If $F \neq A$, then it is possible to construct (in many ways) a subgroup $W$ of $A$ such that $F \leqq W<A$ and such that every element in $A / W$ is of finite order. But every endomorphism with kernel $W$ would map $A$ into $F$. Such an endomorphism cannot then be idempotent, a contradiction which proves that $F=A$, that is, that every element in $A$ is of finite order.

Denote by $P$ the subgroup of $A$ which consists of all the elements in $A$ whose order is a factor of the given prime number $p$. Then there exists an idempotent endomorphism $\epsilon$ of $A$ whose kernel is $P$. Since 1 is the cross cut of $P$ and $A^{\epsilon}$, it is impossible that $A^{\epsilon}$ contains elements of order $p$. Thus 1 is the only element of order a power of $p$ in $A^{e}$. Since $A / P$ and $A^{\epsilon}$ are isomorphic groups, it follows that $P$ contains all the elements of order a power of $p$ in $A$, and this fact implies that the orders of the elements in $A$ are square free, as we intended to prove.

THEOREM 5. If the group $G$ is totally retractable, then it has the following properties:

(a) Every quotient group of $G$ is totally retractable.

(b) Every subgroup of the center of $G$ is a direct factor of $G$.

\footnotetext{
${ }^{3}$ See, for example, Baer [2, chap. II, \$1].
} 
(c) The commutator subgroup $G^{\prime}$ of $G$ is generated by the commutators of the form $x^{-1} y^{-1} x y$ for $x$ in $G$ and $y$ in $G^{\prime}$.

PRoof. Proposition (a) is an almost immediate inference from the fact that every normal subgroup of the quotient group $G / N$ has the form $M / N$ for $M$ a normal subgroup of $G$. To prove (b) consider a subgroup $S$ of the center of $G$. Then $S$ is a normal subgroup of $G$. Hence there exists an idempotent endomorphism $\epsilon$ of $G$ whose kernel is $S$. Since the elements in $S$ commute with every element in $G$, it follows that $G$ is the direct product of $S$ and $G^{e}$. To prove (c) denote by $G^{\prime \prime}$ the subgroup generated by the commutators $x^{-1} y^{-1} x y$ for $x$ in $G$ and $y$ in $G^{\prime}$. Clearly $G^{\prime \prime} \leqq G^{\prime}$ and $G^{\prime \prime}$ is a normal subgroup of $G$. It follows from (a) that $G / G^{\prime \prime}$ is totally retractable. Since $G^{\prime} / G^{\prime \prime}$ is part of the center of $G / G^{\prime \prime}$, it follows from (b) that $G^{\prime} / G^{\prime \prime}$ is a direct factor of $G / G^{\prime \prime}$. But $G^{\prime} / G^{\prime \prime}$ is the commutator subgroup of $G / G^{\prime \prime}$, and a commutator subgroup which is an abelian direct factor is necessarily the identity. Hence $G^{\prime}=G^{\prime \prime}$, completing the proof.

Proposition (b) and (c) of Theorem 5 imply in particular the fact that nilpotent, totally retractable groups are always abelian. On the other hand already the non-abelian group of order 6 provides an example of a soluble, non-abelian group which is totally retractable.

REINHOLD BAER

\section{BibliogRAPHY}

1. Die Kompositionsreihe der Gruppe aller eineindeutigen Abbildungen einer unendlichen Menge auf sich, Studia Mathematica vol. 5 (1934) pp. 15-17.

2. Representations of groups as quotient groups. I, Trans. Amer. Math. Soc. vol. 58 (1945) pp. 295-347.

R. D. CARMichael

1. Introduction to the theory of groups of finite order, Boston, 1937.

J. Schreier and S. Ulam

1. Über die Permutationsgruppe der natïrlichen Zahlenfolge, Studia Mathematica vol. 4 (1933) pp. 134-141.

2. Über die Automorphismen der Permutationsgruppe der natibrlichen Zahlenfolge, Fund. Math. vol. 28 (1937) pp. 258-260.

H. ZASSENHAUS

1. Lehrbuch der Gruppentheorie, vol. 1, Hamburger mathematische Einzelschriften, no. 21, 1937.

UNIVERSITY OF ILLINOIS 